\title{
Wireless operations for 13.56-MHz band RFID tag using amorphous oxide TFTs
}

\author{
Hiroaki Ozaki ${ }^{\text {a)}}$, Tetsufumi Kawamura, Hironori Wakana, \\ Takanori Yamazoe, and Hiroyuki Uchiyama
}

Hitachi, Ltd., Central Research Laboratory, 1-280, Higashi-koigakubo, Kokubunnjishi, Tokyo 185-8601, Japan

a)hiroaki.ozaki.yu@hitachi.com

\begin{abstract}
This paper presents an RFID chip for $13.56-\mathrm{MHz}$ band communication fabricated on a glass substrate by using amorphous InGa-Zn-O thin-film transistors. Low driving-voltage logic circuits were achieved with a small Vth, a high field effect mobility of $15 \mathrm{~cm}^{2} / \mathrm{Vs}$ and "active load" inverters that had small consumption currents. The RFID tag was successively driven by $13.56-\mathrm{MHz}$ wireless input.
\end{abstract}

Keywords: Oxide-Semiconductor, a-IGZO, TFT, RFID, 13.56-MHz Classification: Electron devices, circuits, and systems

\section{References}

[1] K. Finkenzeller, Book RFID HANDBOOK, John Wiley \& Son, Chichester, 1999.

[2] M. Böhm, A. Ullmann, D. Zipperer, A. Knobloch, W. H. Glauert, and W. Fix, "Printable Elecronics for Polymer RFID Applications," Proc. ISSCC 2006, San Francisco, pp. 1034-1041, Feb. 2006.

[3] R. Blache, J. Krumm, and W. Fix, "Organic CMOS circuits for RFID applications," Proc. ISSCC 2009, San Francisco, pp. 208-209, 209(a), Feb. 2009.

[4] K. Myny, M. J. Beenhakkers, N. A. J. M. van Aerle, J. Genoe, W. Dehaene, and P. Heremans, "Robust digital design in organic electronics by dual-gate technology," Proc. ISSCC 2010, San Francisco, pp. 140-141, Feb. 2010.

[5] H. Yin, S. Kim, C. J. Kim, J. C. Park, I. Song, S. Kim, S. Lee, and Y. Park, "Bootstrapped Ring Oscillator with Propagation Delay Time below $1.0 \mathrm{nsec} /$ stage by Standard $0.5 \mu \mathrm{m}$ Bottom-Gate Amorphous $\mathrm{Ga}_{2} \mathrm{O}_{3}$ $\mathrm{In}_{2} \mathrm{O}_{3}-\mathrm{ZnO}$ TFT Technology," IEDM Tech. Dig., pp. 81-84, 2006.

[6] H. Yin, S. Kim, J. Park, I. Song, S. Kim, J. Hur, S. Park, S. Jeon, and C. J. Kim, "High Performance Low Voltage Amorphous Oxide TFT Enhancement/Depletion Inverter through Uni-/Bi-Layer Channel Hybrid Ingegration," IEDM Tech. Dig., pp. 199-202, 2009.

[7] T. Kawamura, H. Uchiyama, S. Saito, H. Wakana, T. Mine, M. Hatano, $\mathrm{K}$. Torii, and T. Onai " $1.5-\mathrm{V}$ operating fully-depleted amorphous oxide thin film transistors achieved by $63-\mathrm{mV} /$ dec subthreshold slope," IEDM Tech. Dig., pp. 77-80, 2008.

[8] T. Kawamura, H. Wakana, K. Fujii, H. Ozaki, K. Watanabe, T. Yamazoe, H. Uchiyama, and K. Torii, "Oxide TFT Rectifier Achieving 13.56-MHz 
Wirelss Operation with DC Output up 12 V," IEDM Tech. Dig., pp. 140141, Feb. 2010.

[9] J. Heo, J. Kim, S. Choi, K. S. Park, C. D. Kim, Y. K. Hwang, and I. J. Chung, "17.4L: Late-News Paper: Contact Resistance and Process Integration Effects on High-Performance Oxide TFTs with SolutionDeposited Semiconductor and Gate Dielectric Layers," SID Symposium Dig., vol. 41, no. 1, pp. 241-244, 2010.

[10] J. Seon, M. Ryu, K. Park, I. Kee, Y. Lee, B. Koo, and S. Y. Lee "SolutionProcessed Indium Zinc Oxide Thin Film Transistors for AMOLED Operation," IDW, pp. 1693-1696, 2009.

\section{Introduction}

The range of applications of radio frequency identification (RFID) technologies [1] has been spreading in recent years. RFIDs are used in systems such as those for entering and leaving management systems, in book management systems in libraries, and in supply chain management systems. However, their use is still limited despite their high potential due to the problem with cost. Therefore reducing the cost of constructing tags is the key to expand their applications.

Non-silicon-based semiconductor devices using oxides and/or organics have recently been focused on for their high cost efficiency, flexibility, and transparency. Thus, these technologies are candidates that are expected to reduce the cost of fabricating tags.

RFIDs consisting of organic thin film transistors (TFTs) have been studied $[2,3,4]$ and they have achieved wireless operations of a few kilohertz. However, they need high input power and have difficulties with enhancing the speed of operations.

Amorphous In-Ga-Zn-O (a-IGZO) TFTs have high mobility, which enables high-speed operation $[5,6]$ with low-power consumption. They can also be fabricated with low-temperature and low-cost processes. In addition, these processes are compatible with the roll-to-roll process.

Several groups have formed thin-film oxide semiconductors with solution processes and fabricated TFTs by using these $[9,10]$. These solution processes should enable us to form ultra-low-cost printable RFID tags; however, compared to sputtering processes, solution processes still have issues in achieving high-performance TFTs and reducing process temperatures.

An RFID chip constructed from a-IGZO TFTs fabricated with a sputtering process is presented in this paper to expand RFID applications by achieving low-cost oxide tags. Our demonstrations of wireless operations with tags are also discussed. Oxide tags are expected to be candidates for functional tags such as those that are printable, flexible, and transparent in addition to tags that are low cost. 


\section{RFID chip architecture}

The RFID chip architecture and up-link (tags to reader) signal protocol are outlined in Fig. 1. The RFID chip consists of RF circuits (a half-wave rectifier, a shunt regulator, a load modulator, and a reset circuit) and logic circuits (a ring oscillator, a read only memory (ROM), a memory driver, and an encoder). We used a total of 1026 TFTs in the chip.

Fig. 1(b) is the circuit diagram for a simple RFID tag (consisting of a chip and coil antenna) circuit model. When RF power is supplied to the coil antenna through the magnetic field coupling between the tag coil and reader coil, the half-wave rectifier, which is constructed from a saturated load n-type TFT and a smoothing capacitor, produces DC supply voltage. To prevent excessive DC voltage been applied to logic circuits, there is a shunt regulator, which dumps unnecessary current, behind the rectifier. The reset circuit is also put directly behind the rectifier similarly to that in the regulator. The

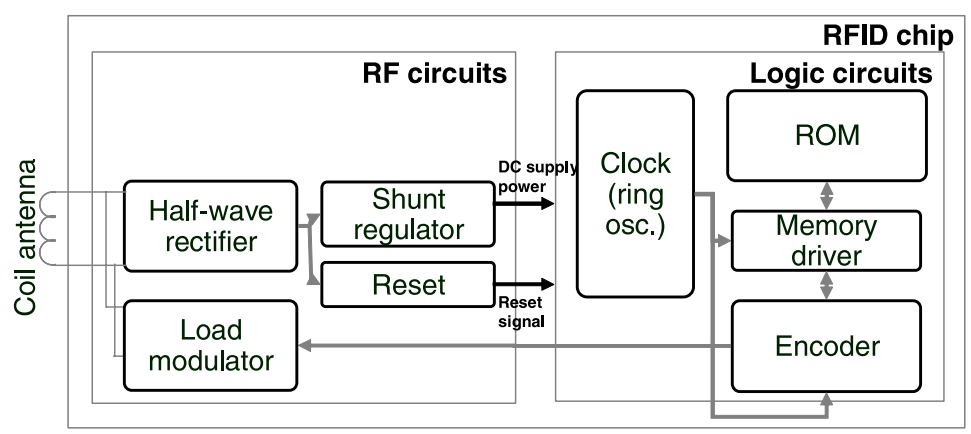

(a)

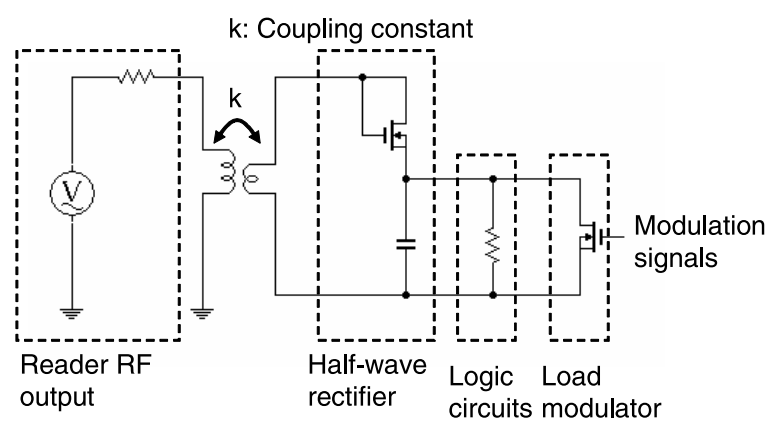

(b)

\begin{tabular}{|c|c|c|}
\hline Preamble & Unique word & ROM data \\
\hline \multicolumn{3}{|c|}{ 几几几几几几几 } \\
\hline 8 clock cycle & $\begin{array}{l}4 \text { clock cycle } \\
\text { Inverted first bit } \\
\text { of data signal }\end{array}$ & $\begin{array}{l}\text { NRZ encoding } \\
\text { ROM data }\end{array}$ \\
\hline
\end{tabular}

(c)

Fig. 1. (a) RFID chip architecture, (b) RFID tag circuit model, and (c) tag signal protocols. 
reset circuit outputs "high" voltage when the DC voltage is lower than the threshold voltage and outputs "low" voltage when the DC supply voltage is higher than the threshold voltage. The reset off signal, vis., "low" voltage, triggers the operations of the logic circuits. The ring oscillator (consisting of 11 serial inverters) in the logic circuits generates clock signals, and then the encoder generates the tag response signals shown in Fig. 1 (c).

The tag response signals start with a preamble signal, which consists of eight cycles of tag internal clock. Then, a unique word signal, which is an inverted signal of the first ROM data lasting for four clock cycles, and the ROM data signals follow.

The four-bit data of the ROM that is serialized by the memory driver and then sent to the encoder. The data are encoded by a non-return-to-zero (NRZ) code and then sent to the load modulators. As shown in Fig. 1 (b), a TFT whose drain/source ports are directly connected to the DC supply is used as the load modulator. The load modulator changes the DC supply voltage according to the encoded signals inputted into its gate by switching on and off. When the DC supply changes, the power consumption by the tag fluctuates, and then these fluctuations are conveyed to the reader coil through a magnetic field. Thus, an RFID reader detects fluctuations, and then decodes them into tag response signals.

\section{3 a-IGZO TFT and active load inverter}

There is a cross section of a bottom-gate/top-contact a-IGZO TFT in Fig. 2. To achive low driving voltage for logic circuits, fully-depleted type TFTs are employed [7]. The fabrication process has been described in our previous paper [8].

Fig. 2 (b) plots the drain-source current (Ids) corresponding to the gate voltage (Vgs). A small threshold voltage (Vth) is achieved, and a field effect mobility of $15.1 \mathrm{~cm}^{2} / \mathrm{Vs}$ can be derived from Fig. 2 (b).

There is a circuit diagram of an active load inverter circuit in Fig. 2 (c). The inverter has load (top) and switch (bottom) transistors. The gate of the load transistor is connected to the "OUT" port. When a "high" state voltage is applied to the gate of the switch transistor, the resistance of the drain-source of the switch transistor decreases, and then the "OUT" port enters a "low" state. Because the "OUT" port and gate of the load transistor are connected, the load transistor turns off, and has high resistance. However, when the gate of the switch transistor becomes "low", the "OUT" port exports a "high" state, and the load transistor turns on. Thus, the load transistor changes its resistance actively according to the "IN" port state.

The Vin-Vout characteristics and consumption currents of the inverter are plotted in Fig. $2(\mathrm{~d})$. The inverter has a good on-off ratio, and a small consumption currents of $1 \mathrm{nA}$. 


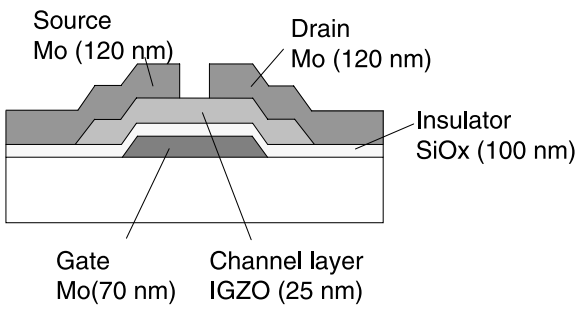

(a)

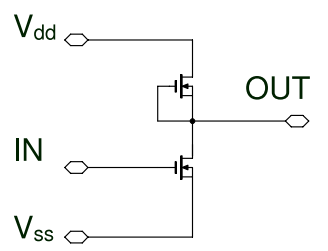

(c)

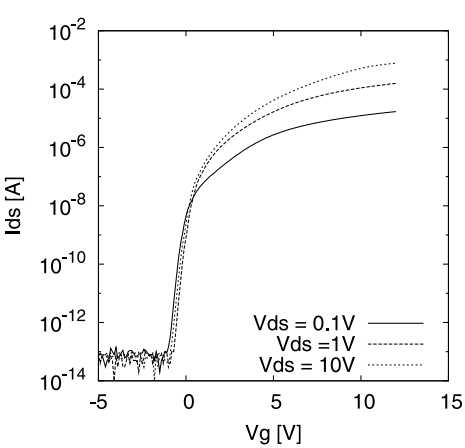

(b)

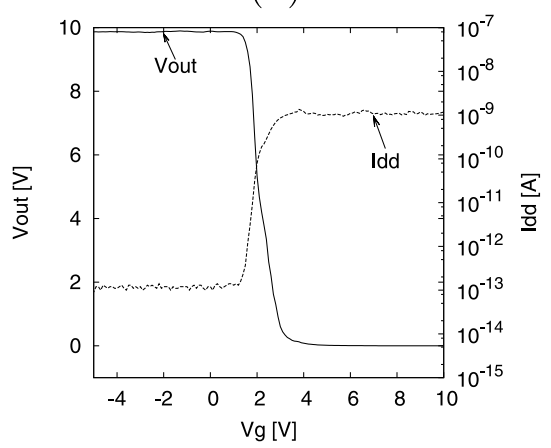

(d)

Fig. 2. (a) Schematic of the oxide TFT, (b) Vg-Ids characteristics, (c) inverter circuit, and (d) Vin-Vout characteristics.

\section{Results and discussion}

There is a photograph of the RFID chips fabricated on a 3-inch glass substrate in Fig. 3 (a). The chips are about $10 \mathrm{~mm}$ long and about $8 \mathrm{~mm}$ wide. As previously mentioned, the output signals from an RFID chip are detected as fluctuations in DC voltage supplied to the logic circuits.

Fig. 3 (b) plots the measured data for a fabricated RFID chip. The preamble, unique word, and ROM data signals can clearly be observed. A pulse generator was connected to the two antenna ports and 13.56-MHz RF power was supplied between the two ports through the wires in the measurements. The supply started at $0 \mathrm{sec}$ and the DC supply voltage, viz., the output from the rectifier, rose to $5 \mathrm{~V}$. The tag responses started at $0.3 \mathrm{sec}$ and were observed as voltage drops (note that the tag response signals in our RFID tag were the inverse of the encoder's output). The internal clock frequency of the tag was estimated to be $15 \mathrm{~Hz}$ from the cycles of the preamble signal. The unique word signal, which is a four clock cycles of "low" state in the encoder's output, was observed as "high" DC voltage. The ROM data signals started at $1.3 \mathrm{sec}$ with the "high" state (i.e., a voltage drop) and was read as "1 0 10 ". At the end of the ROM data signals, a short-term voltage drop was found, and it was considered to have been caused by logic circuit delay. The deviation in the time length of the data signals might affect the reading success ratio. However, the dispersion of the clock cycle length was sufficiently small to read. The average time length in the high state was shorter that in 


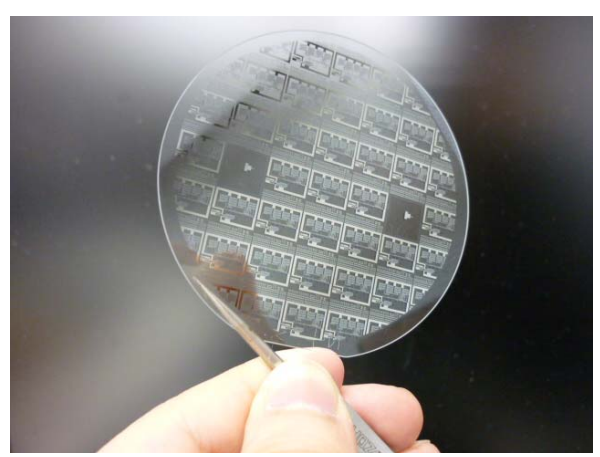

(a)

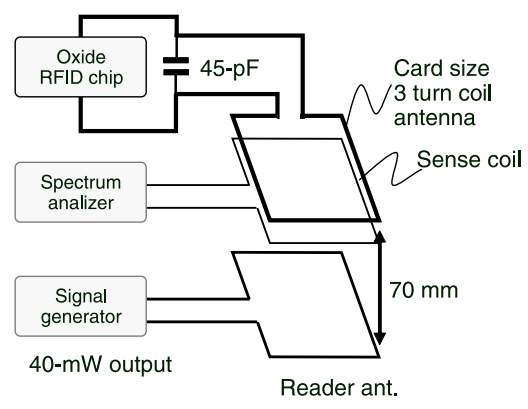

(c)

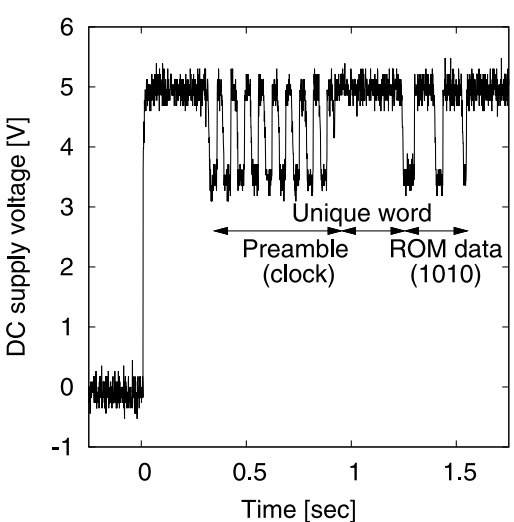

(b)

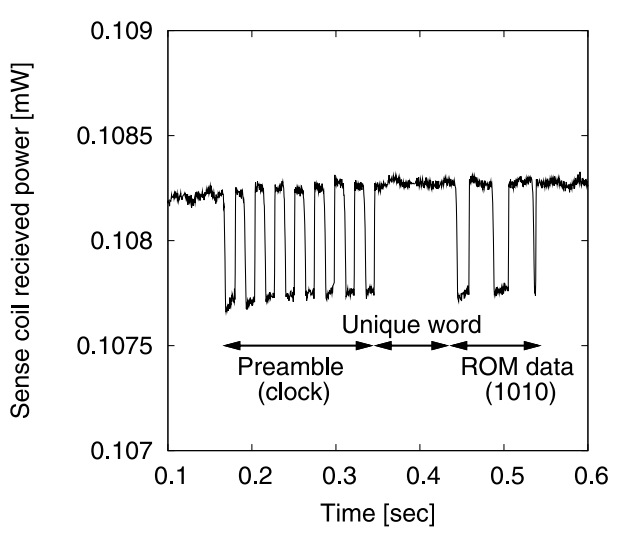

(d)

Fig. 3. (a) Fabricated chips, (b) DC supply voltage of RFID chip, (c) wireless measurement system, and (d) RF power detected by sense coil.

than that in the low state due to charging delay in the load modulator TFT. We found the RFID chip could be operated at a low supply DC supply voltage of $5 \mathrm{~V}$ from the wired measurements.

Fig. 3 (c) outlines the a wireless measurement system, where the a-IGZO RFID chip is connected to a 45-pF resonance capacitor and a card sized threeturn coil antenna to achieve an RFID tag. A signal generator is connected to a commercial RFID reader antenna and the three-turn coil is $70 \mathrm{~mm}$ above the reader. There is a sense coil under the three-turn coil to detect the fluctuations in RF power. The RFID tag is wirelessly driven with $13.56-\mathrm{MHz}$ input from the reader. The input power of $40 \mathrm{~mW}$ is supplied from the signal generator which is the minimum power of the commercial reader we used. As seen in Fig. 3(d), the tag response signals were successfully confirmed despite the low RF input. The tag internal clock frequency was found to be $50 \mathrm{~Hz}$ from wireless measurements, which was three times faster than that in the wired measurements. The resistance and capacitance of the measuring probe were considered to have caused clock frequency delay in the wired measurements. The clock frequency of $50 \mathrm{~Hz}$ was high enough for detecting fluctuating signals of the tag with readers. However, the higher frequency is 
required to adapt to international standards for $13.56-\mathrm{MHz}$ band RFIDs.

\section{Summary}

An a-IGZO RFID chip for 13.56-MHz-band communication was successfully developed. It consisted of RF and logic circuits and they were constructed of 1026 TFTs. The logic circuits generated tag response signals with a low DC supply voltage of $5 \mathrm{~V}$. The a-IGZO RFID tag was wirelessly driven by 40-mW input with a communication distance of $70 \mathrm{~mm}$.

\section{Acknowledgments}

The authors wish to thank Messrs. K. Watanabe, K. Torii, and T. Onai for their continued support and encouragement throughout out research. 